\title{
Erratum to: Discovery of Chrysoeriol, a PI3K-AKT-mTOR Pathway Inhibitor with Potent Antitumor Activity against Human Multiple Myeloma Cells In Vitro
}

Yang YANG, Xiaoxi ZHOU, Min XIAO, Zhenya HONG, Quan GONG, Lijun JIANG, Jianfeng ZHOU

Department of Hematology, Tongji Hospital, Tongji Medical College, Huazhong University of Science and Technology, Wuhan 430030, China

(C) Huazhong University of Science and Technology 2020

Current Medical Science

https://link.springer.com/article/10.1007/s11596-010-0649-4

30(6):734-740, 2010

The authors examined the original data of their work and noticed a misuse of the image of the bands of AKT and actin in fig. 2A (as shown below on the upper panel). The error occurred during the preparation of the manuscript, mainly due to the wrongly labeling of image items or folders inadvertently. The correct one is shown in fig. 2 on the lower panel. The authors sincerely apologize for the mistake and confirm that the change does not affect the scientific conclusion of the published work.

A

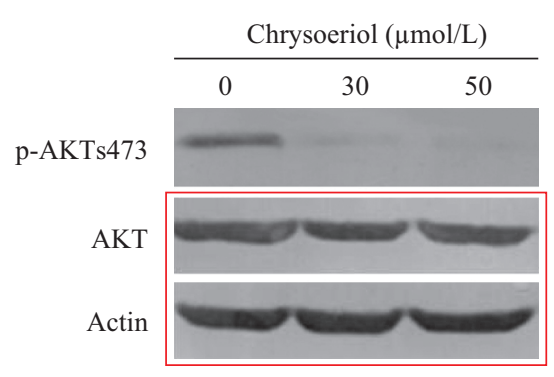

A

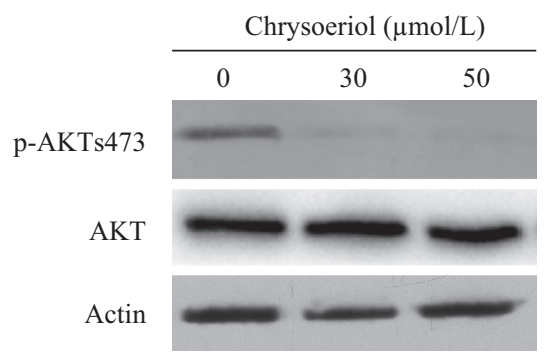

B

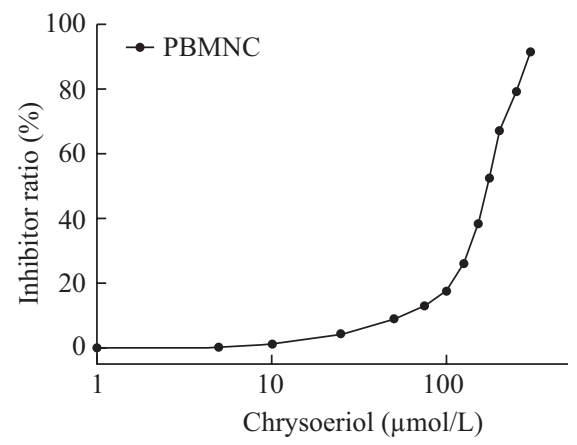

B

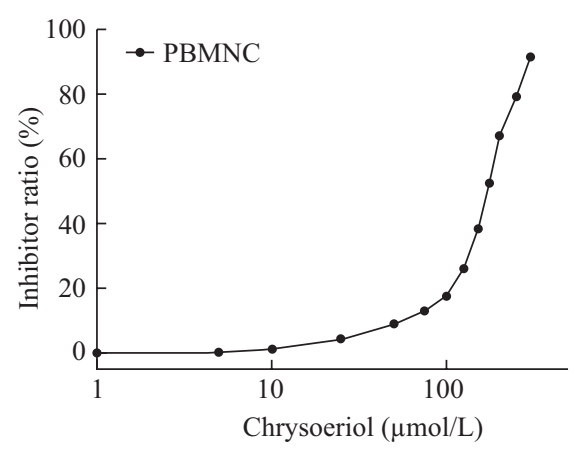

Fig. 2 Effects of chrysoeriol on phosphorylated AKT levels and proliferation of PBMNCs from healthy donors

A: Effect of Chrysoeriol on p-AKT (s473) and AKT protein levels; B: the curve of inhibition rate of PBMNCs against chrysoeriol. $\mathrm{IC}_{50}$ concentration of PBMNCs was about $175 \mu \mathrm{mol} / \mathrm{L}$. The red rectangle indicates the error. 